\title{
Multifractal Characterization of the Autonomous Nervous System during Prolonged Coronary Artery Occlusion
}

\author{
Rudys Magrans, Pedro Gomis, Member, IEEE, Pere Caminal, Member, IEEE and Galen Wagner
}

\begin{abstract}
We assess the autonomic nervous system response during prolonged percutaneous transluminal coronary angioplasty (PTCA) by heart rate variability analysis using multifractal indices. These indices are also used to evaluate the effects of the PTCA at different arteries. The indices augmented significantly during transient ischemia and reperfusion periods indicating an increase of multifractal degree and a decrease of the long-range dependence on heartbeat fluctuations. This indicates that significant multifractal complex reactions of autonomic control of the heart rate occurred during coronary artery occlusions.

Key words: multifractal analysis, heartbeat fluctuations, myocardial ischemia, coronary artery occlusion
\end{abstract}

\section{INTRODUCTION}

$\mathrm{H}$ EART rate control is perturbed by alterations in neuroautonomic function in a number of important clinical syndromes, including sudden cardiac death, congestive failure, physiologic aging, etc., which are associated with a loss of the normal fractal complexity of heart rate variability (HRV) dynamics [1].

In the presence of coronary obstruction, an imbalance occurs between myocardial oxygen supply and demand, provoking myocardial ischemia. The percutaneous transluminal coronary angioplasty (PTCA) has been a technique used to open blocked coronary arteries caused by atherosclerotic plaques within the coronary arteries and restoring arterial blood flow to the myocardium. PTCA has been a good model to evaluate myocardial ischemia.

Several experimental studies have demonstrated that coronary occlusion activates afferent cardiac sympathetic nerves, driving a sympathetic reflex, and can modify the activity generated by intrinsic cardiac neurons [2]. Some clinical evaluations of HRV during PTCA have been reported [3]-[5]. These studies use standard time and frequency-domain methods to evaluate the influence of sympathetic and parasympathetic activity or its balance produced by different occlusions during PTCA. Previous

Manuscript received April 21, 2009. This work was supported in part by CICYT grant TEC2007-63637 from the Spanish government. The first author was supported by the Grant 2008FI_B 00651 from the Comissionat per a Universitats i Recerca del Departament d'Innovació, Universitats i Empresa of the Government of Catalonia and the European Social Fund.

R. Magrans, P. Gomis, and P. Caminal are with the Centre de Recerca en Enginyeria Biomèdica (CREB), Dept. ESAII, Universitat Politècnica de Catalunya (UPC), and CIBER de Bioingeniería, Biomateriales y Nanomedicina (CIBER-BBN), c/ Pau Gargallo 5, 08028, Barcelona, Spain (e-mail: \{rudys.magrans, pedro.gomis, pere.caminal\}@upc.edu).

G. Wagner is with Duke University Medical Center, Durham, North Carolina, USA (e-mail: galen.wagner@duke.edu). studies have already evaluated the autonomic nervous system (ANS) behavior during prolonged PTCA by HRV analysis using fractal-like indices [6] and multiscale information analysis (MIA) of HRV has taken into account entropy rates [7]. Recently, a method that combines surrogate data analysis and information-theoretic measures to quantify the changes in heart rate dynamics during myocardial ischemia induced by PTCA has been developed [8].

The multifractality has been reported in heart rate fluctuations of healthy individuals [9]. Lately, the multifractal analysis has become an useful tool to detect multifractality and long-range correlation in heartbeat fluctuations for the diagnosis of heart failure [9], [10].

The aim of this study is to assess multifractal characteristics of the autonomic control of the cardiac rhythm during ischemic events provoked by long PTCA procedures.

\section{MATERIALS AND METHODS}

\section{A. Study population}

This study was based on patients from the Staff3 database undergoing elective PTCA in the catheterization laboratory at the Charleston Area Medical Center, West Virginian, USA [11]. Continuous high-resolution ECGs were acquired at $1 \mathrm{kHz}$ sample frequency, with $0.6 \mathrm{mV}$ amplitude resolution. Nine lead signals (standard I, II and III, and precordial V1-V6) were stored on hard disk for subsequent analysis.

Fifty five patients who met the following criteria were considered in the study: (a) no history of coronary bypass surgery, without left or right ventricular hypertrophy; (b) no evidence of right bundle branch block; (c) QRS duration less than $120 \mathrm{~ms}$ measured on a previous control ECG; (d) no clinical or ECG evidence of previous myocardial infarction; and (e) artery occlusion duration of at least 3 minutes.

Patients were classified according to the occluded coronary artery in three groups: right coronary artery (RCA) with 25 patients; left anterior descending (LAD) coronary artery with 20 patients; and left circumflex coronary artery (LCX) with 10 patients.

The balloon inflation period ranged from 3 to $5 \mathrm{~min}$. The occlusion period was considerably longer than that normally used for coronary angioplasty procedures because the treatment protocol included a single prolonged occlusion instead of a series of brief occlusions. HRV analysis were 
performed using 3 minutes of R-R signal from the preinflation ECG (pre-PTCA), the first 3 minutes (PTCA1) and last 3 minutes (PTCA2) of the ECG during PTCA, and approximately 3 minutes after the PTCA procedure finished (post-PTCA). Before, the R-R time series was resampled at $5 \mathrm{~Hz}$ using cubic splines interpolation with the purpose to obtain uniformly sampled data.

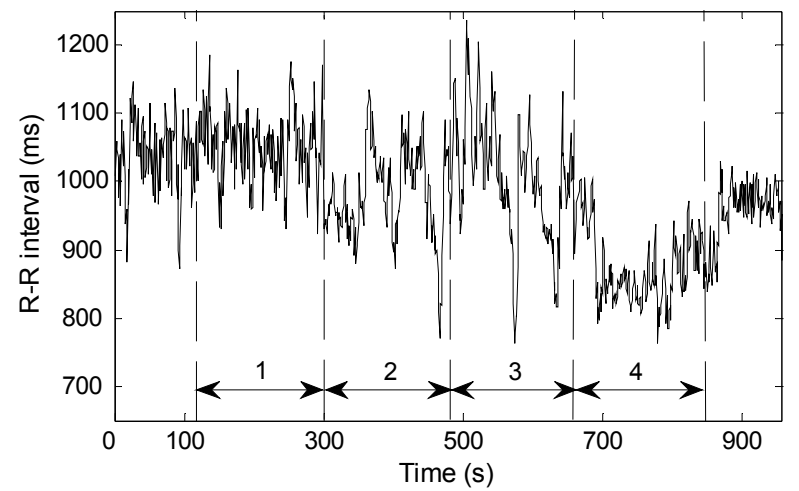

Fig. 1. R-R signal of one of the studied patients with LAD-MID occlusion. The periods before inflation (1), during balloon inflation (2 - 3), and after the occlusion (4) are shown.

\section{B. Multifractal analysis}

The simplest method of multifractal analysis is based on multifractal formalism [12]. Unfortunately, it is not applicable for nonstationary time series. Recently, Kantelhardt et al.[13] has proposed the multifractal detrended fluctuation analysis (MF-DFA), an alternative approach based on a generalization of the detrended fluctuation analysis (DFA).

The original $\mathrm{R}-\mathrm{R}$ time series, from which its average value has been subtracted, is integrated as:

$Y(i) \equiv \sum_{k=1}^{i}\left[R R(k)-R R_{m}\right], \quad i=1, \ldots, N$.

where $R R(k)$ is the $k$ th distance between consecutive beats, being $N$ the size of the R-R signal, and $R R_{m}$ the average value over the recording. The integrated signal $Y(i)$ is divided into $N_{s}=\operatorname{int}(N / s)$ nonoverlapping windows $v$ of equal length $s$ starting from both the beginning and the end of the integrated signal, thereby, a total of $2 N_{s}$ windows are obtained. In each window a $l$ th order polynomial $P_{v}$ is fitted to $Y(i)$ in the least-squares sense, representing the trend in that window. Here, we used a quadratic order polynomial (conventionally called MF-DFA2) because it has the capacity to eliminate trends of second order in the integrated signal (or, order 1 in the original R-R time series). Then determine the variance,

$F^{2}(s, v)=\frac{1}{s} \sum_{i=1}^{s}\left\{Y[(v-1) s+i]-P_{v}(i)\right\}^{2}$

for each window $v=1, \ldots, N_{s}$ and

$F^{2}(s, v)=\frac{1}{s} \sum_{i=1}^{s}\left\{Y\left[N-\left(v-N_{s}\right) s+i\right]-P_{v}(i)\right\}^{2}$ for $v=N_{s}+1, \ldots, 2 N_{s}$.

In order to scan the degree of contribution of a certain window to fluctuations of a time series, a qth order fluctuation function is defined as follows:

$$
F_{q}(s) \equiv\left\{\frac{1}{2 N_{s}} \sum_{v=1}^{2 N_{s}}\left[F^{2}(s, v)\right]^{q / 2}\right\}^{1 / q}
$$

where the index variable $q$ can take any real value except zero. When $q=0$ then

$F_{0}(s) \equiv \exp \left\{\frac{1}{4 N_{s}} \sum_{v=1}^{2 N_{s}} \ln \left[F^{2}(s, v)\right]\right\}$.

This fluctuation function reveals power-law scaling for large $s$,

$F_{q}(s) \sim s^{h(q)}$.

The family of scaling exponents $h(q)$ (also called the generalized Hurst exponents) are a decreasing function of $q$ for multifractal signals, while for monofractal signals $h(q)$ is independent of $q$. From $h(q)$ we can directly calculate the the singularity exponent $\alpha$ and the singularity spectrum $f(\alpha)$ using the following relations:

$\alpha=h(q)+q \frac{d h(q)}{d q}$ and $f(\alpha)=q[\alpha-h(q)]+1$.

In our study, we vary the value of $s$ in the range from $s_{\min }=4$ to $s_{\max }=N$, and we used values of $q$ between -5 and 5 .

The $f(\alpha)$ function has a concave downward curvature, its shape and extension contains significant information about the distribution characteristic of the data set. The width of $f(\alpha)$ is defined by the index $\Delta \alpha=\alpha_{\max }-\alpha_{\min }$, which has been used in several studies to evaluate the multifractality degree of $f(\alpha)$ in heart rate dynamics corresponding to healthy and pathologic subjects [9], [10], [14], [15]. Also, the value of the singularity exponent $\alpha_{m}$ for which $f(\alpha)$ is maximum was considered as an index. Through this index we are able to find the exponent $\alpha$ where the singularity spectrum is centered at to measure the long-range correlations on the fluctuations in heartbeat intervals.

\section{Statistical analysis}

A Wilcoxon nonparametric rank-based test was used in order to assess the statistical significance when comparing values of the indexes between different periods. Significant differences were taken into account when $\mathrm{p}<0.05$. All analyses were performed with the SPSS for Windows release 15.0 (SPSS Inc., Chicago, Ill).

\section{RESULTS}

The multifractal indexes summarized in Table I revealed changes associated with ischemia provoked by coronary artery occlusions. Such changes are statistically significant 
when comparing the R-R signals during the majority of the PTCA procedure periods.

The averaged singularity spectrum $f(\alpha)$ for RCA, LAD, LCX studied groups and for the total patients before inflation, during balloon inflation, and after occlusion are represented on Fig. 2. Fig. 3 presents a graphical evolution of the multifractal indices along PTCA procedure.

As we expected, due to the accounted changes on $h(q)$ the width of the $f(\alpha)$ spectra increases significantly during postinflation $(\Delta \alpha=0.68 \pm 0.20)$ with respect to pre-inflation $(\Delta \alpha$ $=0.43 \pm 0.15, \mathrm{p}<0.001)$, and both balloon inflation periods $(0.53 \pm 0.18, \mathrm{p}=0.001 ; 0.49 \pm 0.17, \mathrm{p}<0.001)$ on RCA occlusion. As we can see in Fig. 2, the differences in $\Delta \alpha$ for
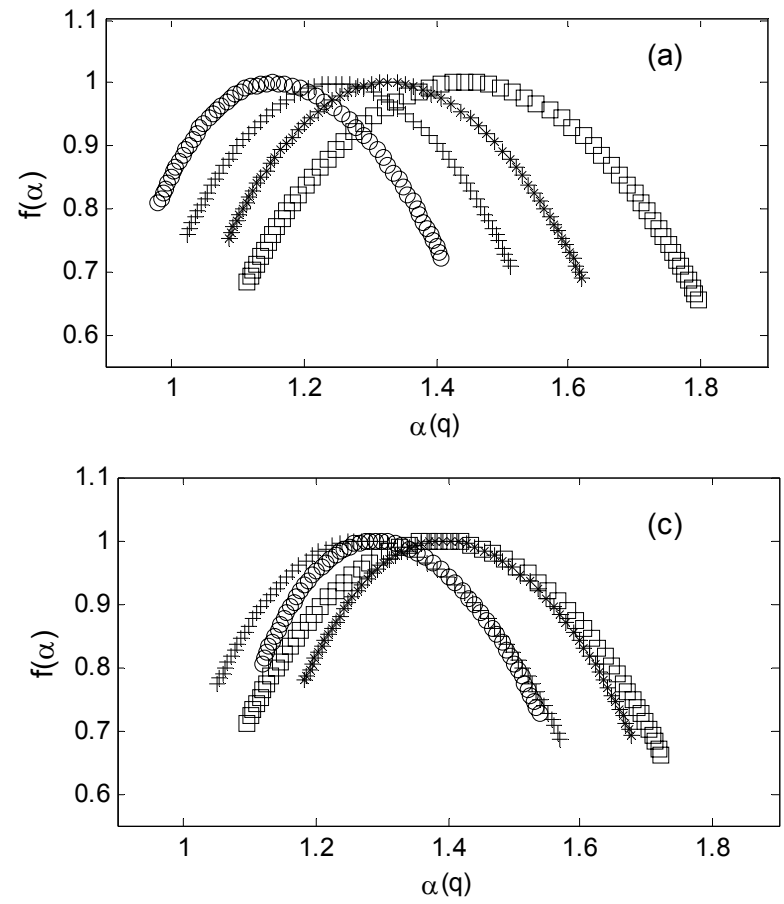

RCA group are larger than the differences found for LAD (pre-PTCA vs. post-PTCA, $p=0.028$; PTCA1 vs. postPTCA, $\mathrm{p}=0.028$ ) and LCX (pre-PTCA vs. post-PTCA, $\mathrm{p}=$ 0.007 ; PTCA1 vs. post-PTCA, $\mathrm{p}=0.028$ ) groups. No significant differences were found in $\Delta \alpha$ between the last $180 \mathrm{~s}$ of occlusion and post-occlusion when we studied LAD and LCX groups for separate. No significant differences were found in $\Delta \alpha$ between PTCA periods for any artery group either. For all occluded arteries, post inflation period showed statistically significant increase $(\mathrm{p}<0.001)$ in $\Delta \alpha$ compared to before and during the balloon occlusion. Besides, significant differences are present between PTCA values and pre-PTCA $(\mathrm{p}=0.005$ and $\mathrm{p}=0.024)$.
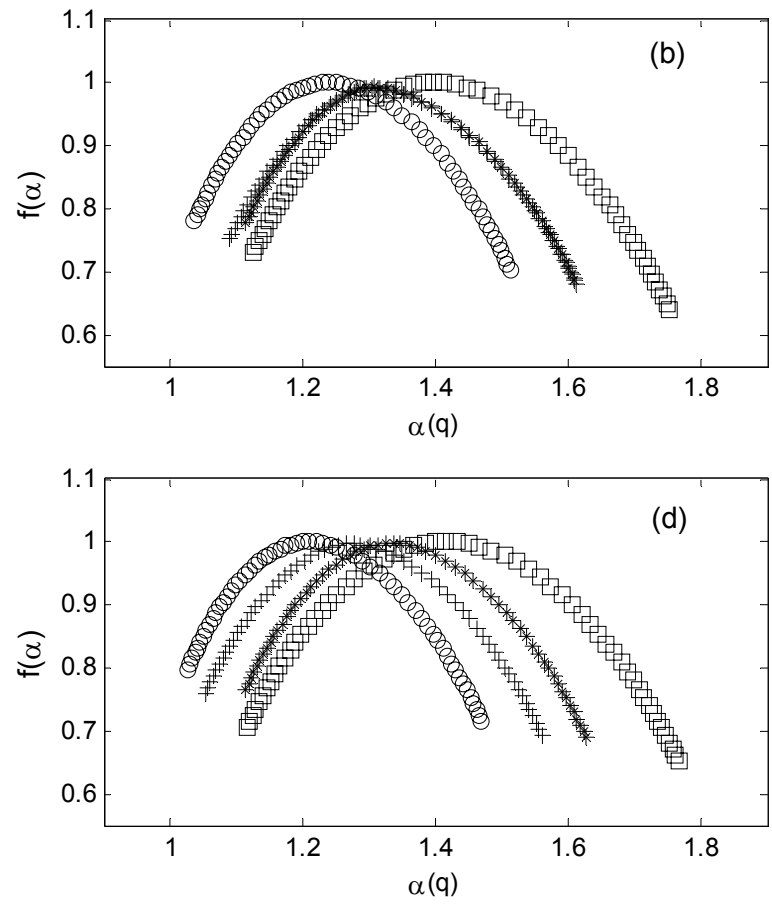

Fig. 2. The averaged singularity spectrum $\mathrm{f}(\alpha)$ for the studied periods: pre-PTCA $(\circ)$, PTCA1 $(*)$, PTCA2 (+), and post-PTCA ( $\square)$ on the (a) RCA, (b) LAD, (c) LCX groups of patients classified according to the occluded coronary artery, and (d) for all the patients.

Table I

Mean \pm standard deviation values and p-value for Wilcoxon nonparametric rank-based test

\begin{tabular}{|c|c|c|c|c|c|c|c|c|c|c|c|}
\hline \multirow{2}{*}{ Groups } & \multirow{2}{*}{ Index } & \multicolumn{4}{|c|}{ Studied period } & \multicolumn{6}{|c|}{$p$-value } \\
\hline & & pre-PTCA & PTCA1 & PTCA2 & post-PTCA & $p^{*}$ & $\mathrm{p}^{* *}$ & $\mathrm{p}^{+}$ & $\mathrm{p}^{++}$ & $p^{\dagger}$ & $\mathrm{p}^{\ddagger}$ \\
\hline \multirow{2}{*}{$\begin{array}{c}\text { RCA } \\
\text { (25 pts.) }\end{array}$} & $\Delta \alpha$ & $0.43 \pm 0.15$ & $0.53 \pm 0.18$ & $0.49 \pm 0.17$ & $0.68 \pm 0.20$ & 0.007 & 0.143 & $<0.001$ & 0.274 & 0.001 & $<0.001$ \\
\hline & $\alpha_{\mathrm{m}}$ & $1.15 \pm 0.13$ & $1.33 \pm 0.25$ & $1.25 \pm 0.18$ & $1.44 \pm 0.27$ & 0.004 & 0.030 & $<0.001$ & 0.014 & 0.045 & 0.002 \\
\hline \multirow{2}{*}{$\begin{array}{c}\text { LAD } \\
(20 \text { pts. }) \\
\end{array}$} & $\Delta \alpha$ & $0.48 \pm 0.15$ & $0.50 \pm 0.15$ & $0.53 \pm$ & 0.63 & 0.538 & 0.313 & 0.028 & 0.356 & 0.028 & 0.135 \\
\hline & $\alpha_{\mathrm{m}}$ & $1.24 \pm 0.20$ & $1.32 \pm 0.23$ & $1.32 \pm 0.21$ & $1.40 \pm 0.14$ & 0.076 & 0.126 & 0.006 & 0.943 & 0.167 & 0.086 \\
\hline \multirow{2}{*}{$\begin{array}{c}\text { LCX } \\
(10 \text { pts. }) \\
\end{array}$} & $\overline{\Delta \alpha}$ & $0.42 \pm 0.11$ & $0.49 \pm 0.14$ & $0.52 \pm 0.19$ & 0.63 & 0.169 & 0.114 & 0.007 & 0.508 & 0.028 & 0.114 \\
\hline & $\alpha_{\mathrm{m}}$ & $1.29 \pm 0.16$ & $1.39 \pm 0.18$ & $1.27 \pm 0.19$ & $1.39 \pm 0.19$ & 0.139 & 0.878 & 0.508 & 0.009 & 0.799 & 0.139 \\
\hline \multirow{2}{*}{$\begin{array}{c}\text { ALL } \\
(55 \text { pts. })\end{array}$} & $\Delta \alpha$ & $0.44 \pm 0.14$ & $0.51 \pm 0.16$ & $0.51 \pm 0.17$ & $0.65 \pm 0.18$ & 0.005 & 0.024 & $<0.001$ & 0.958 & $<0.001$ & $<0.001$ \\
\hline & $\alpha_{\mathrm{m}}$ & $1.21 \pm 0.17$ & $1.34 \pm 0.23$ & $1.28 \pm 0.19$ & $1.42 \pm 0.22$ & $<0.001$ & 0.021 & $<0.001$ & 0.002 & 0.021 & $<0.001$ \\
\hline
\end{tabular}

$\mathrm{p}^{*}$, pre-PTCA vs. PTCA1; $\mathrm{p}^{* *}$, pre-PTCA vs. PTCA2; $\mathrm{p}^{+}$, pre-PTCA vs. post-PTCA; $\mathrm{p}^{++}$, PTCA1 vs. PTCA2; $\mathrm{p}^{\dagger}$, PTCA1 vs. post-PTCA; $\mathrm{p}^{\dagger}$,

PTCA2 vs. post-PTCA

On the other hand, we have found statistically significant changes along PTCA procedure in the singularity exponent $\alpha_{m}$ for which $f(\alpha)$ is maximum. These changes are more evident in RCA occlusions than in other occlusions; and these are significant in all periods compared. In the case of LAD occlusions, a significant increase was observed only in
post-PTCA versus pre-PTCA (from $1.24 \pm 0.20$ to $1.40 \pm$ $0.14 ; p=0.006$ ); whereas for LCX occlusions no significant change was observed during post-PTCA period with respect to before and during occlusion periods. For the entire set of occluded arteries, $\alpha_{m}$ shows significant increases in all periods compared; except when we compared intra-PTCA 
periods, in this case, $\alpha_{m}$ shows a significant decrease (from $1.34 \pm 0.23$ to $1.28 \pm 0.19 ; \mathrm{p}=0.002$ ).

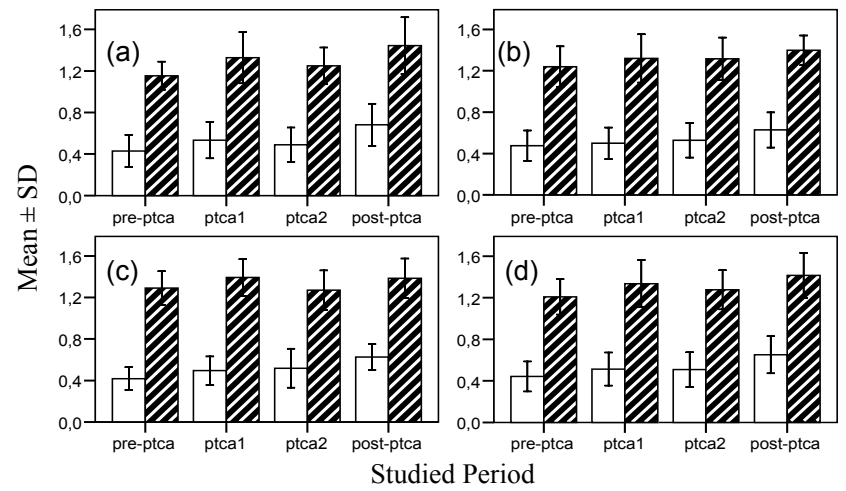

Fig. 3. Evolution of the indices $\Delta \alpha$ (white), $\alpha_{m}$ (cross bar) before, during, and after PTCA procedure on (a) RCA, (b) LAD, (c) LCX occlusions and (d) for all patients.

\section{Discussion AND CONCLUSION}

We showed that the studied R-R series of the Staff3 database has a multifractal complexity due to a larger range of values of singularity exponents $\alpha(q)$ to characterize the fluctuations on the studied heartbeat intervals. The multifractality captured by $\Delta \alpha$ index changes along the PTCA procedure, being weaker before PTCA stages. Beside, we have found that these fluctuations, in general, are more anticorrelated for pre-inflation period than for other periods, because the maximum of $f(\alpha)$ spectra is centered at smaller value of $\alpha_{m}$, whereas for post inflation period the $f(\alpha)$ spectra is centered at larger $\alpha_{m}$ value closer to $\alpha_{m}=1.5$.

Previous works [9], [10] have found a wide range of exponents for healthy subjects, indicating multifractality; and a very narrow range of exponents for pathological conditions, in particular for patients with congestive heart failure, indicating monofractal behavior. These works have also shown that for heart failure, the fluctuations on heart rate intervals are less anti-correlated than for healthy subjects. Recently, Meyer et al.[14] have found different changes in the range of scaling exponents and in the singularity spectrum shape between different pathological conditions (e.g. cardiac transplantation vs. ventricular tachycardia), evidencing the ANS influences on the cardiac rhythm control. On the other hand, Galaska et al.[15] found that the generalized Hurst exponents had a significantly larger range of exponents and the global Hurst exponent was significantly lower in healthy subjects in comparison to patients with systolic dysfunction of left ventricle, when they used MF-DFA algorithm.

Our results have shown an increase in the multifractality of short-term HRV during transient ischemia and reperfusion periods. It could be associated to sympathovagal balance of the ANS towards either a sympathetic or parasympathetic prevalence, and may represent a beneficial adaptive mechanism for increasing coronary flow during myocardial ischemia. There are evidence that balloon inflation and occlusion evokes baroreceptor vagal predominance in response to stretch stimulus of the coronary artery [16].

According to the occluded coronary artery, the observed changes are more notable in patients with RCA occlusions, which may be related with different responses of the ANS according with the site of the ischemia.

Further studies are required to assess multifractal complex reactions in patients with ischemic episodes using larger ECG records, as well as with previous myocardial infarction subjects from the Staff3 database.

\section{REFERENCES}

[1] A. L. Goldberger, "Fractal mechanisms in the electrophysiology of the heart". IEEE Engineering in Medicine and Biology 1992, 11, 47-52.

[2] A. Malliani, J. P. Schwartz, A. Zanchetti, "A sympathetic reflex elicited by experimental coronary occlusion”. Am J Physiol 1969; 217: 703-709.

[3] K. E. J. Airaksinen, K. V. Ylitalo, M. J. Niemelä, K. U. O. Tahvanainen, H. V. Huikuri, "Heart rate variability and occurrence of ventricular arrhythmias during balloon occlusion of a major artery". Am J Cardiol 1999; 83: 1000-1004.

[4] F. Clariá, M. Vallverdú, P. Caminal, "The effects of coronary occlusion location on RR signal". IEEE Eng Med Biol 2002; 21: 5964.

[5] N. Castro, E. Medina, P. Gomis, S. Wong, G. Wagner, "Multiple factor analysis of the autonomous nervous system during PTCA". In Proceedings of the IEEE Engineering in Medicine and Biology, 2005, pp. 940-943.

[6] P. Gomis, P. Caminal, M. Vallverdú, S. Warren, G. Wagner, "Nonlinear dynamic analysis of the cardiac rhythm during transient myocardial ischemia". Biomed Tech 2006; 51:178-181.

[7] J. F. Valencia, M. Vallverdú, P. Gomis, G. Wagner, P. Caminal, "Multiscale information analysis of the autonomous nervous system during myocardial ischemia". Computers in Cardiology 2007; 34:589592.

[8] R. Benitez, et al., "Characterization of the nonlinear content of the heart rate dynamics during myocardial ischemia". Med Eng \& Phys (2009), doi:10.1016/j.medengphy.2008.12.006.

[9] P. Ch. Ivanov, et al., "Multifractality in human heartbeat dynamics". Nature 399, (1999), 461-465.

[10] A. Havlin, et al., "Application of statistical physics to heartbeat diagnosis". Physica A 1999, 274, 99-110.

[11] M. Ringborn, O. Pahlm, G. Wagner, S. Warren, J. Peterson, "The absence of high-frequency QRS changes in the presence of standard electrocardiographic QRS changes of old myocardial infarction". Am. Heart J. 2001, 141 (4) 573-579.

[12] T. C. Halsey, M. H. Jensen, L. P. Kadanoff, I. Procaccia, B. I. Shraiman, "Fractal measure and their singularities: The characterization of strange sets". Phys. Rev. A33 (1986), pp. 11411151.

[13] J. W. Kantelhardt, et al., "Multifractal detrended fluctuation analysis of nonstationary time series". Physica A 2002, 316, 87-114.

[14] M. Meyer, and O. Stiedl, "Self-affine fractal variability of human heartbeat interval dynamics in health and disease". Eur. J. Appl. Physiol. 2003; 90: 305-316.

[15] R. Galaska, et al., "Comparison of wavelet transform modulus maxima and multifractal detrended fluctuation analysis of heart rate in patients with systolic dysfunction of left ventricle". Ann Noninvasive Electrocardiol 2008; 13(2):155-164.

[16] O. Manfrini, G. Morgagni, C. Pizzi, F. Fontana, R. Bugiardini, "Changes in autonomic nervous system activity: spontaneous versus balloon-induced myocardial ischaemia". European Heart Journal (2004) $25,1502-1508$. 